\title{
Dazed and Confused Considered Normal: An Approach to Create Interactive Systems for People with Dementia
}

\author{
Nasim Mahmud ${ }^{1}$, Joël Vogt ${ }^{2}$, Kris Luyten ${ }^{1}$, Karin Slegers ${ }^{3}$, \\ Jan Van den Bergh ${ }^{1}$, and Karin Coninx ${ }^{1}$ \\ 1 Hasselt University - tUL - IBBT, Expertise Centre for Digital Media, \\ Wetenschapspark 2, B-3590 Diepenbeek, Belgium \\ \{nasim.mahmud, kris.luyten, jan. vandenbergh, karin. coninxg\}@uhasselt. be \\ ${ }_{2}$ Department of Informatics, University of Fribourg, Boulevard de Pérolles 90, \\ CH-1700 Fribourg, Switzerland \\ joel.vogt@unifr.ch \\ 3 Centre for User Experience Research (CUO), IBBT / K.U. Leuven \\ Parkstraat 45 bus 3605, B-3000 Leuven, Belgium \\ karin.slegers@soc.kuleuven. be
}

\begin{abstract}
In Western society, the elderly represent a rapidly growing demographic group. For this group, dementia has become an important cause of dependencies on others and causes difficulties with independent living. Typical symptoms of the dementia syndrome are decreased location awareness and difficulties in situating ones activities in time, thus hindering long term plans and activities. We present our approach in creating an interactive system tailored for the needs of the early phases of the dementia syndrome. Given the increasing literacy with mobile technologies in this group, we propose an approach that exploits mobile technology in combination with the physical and social context to support prolonged independent living. Our system strengthens the involvement of caregivers through the patient's social network. We show that applications for people suffering from dementia can be created by explicitly taking into account context in the design process. Context dependencies that are defined in an early stage in the development process are propagated as part of the runtime behavior of the interactive system.
\end{abstract}

\section{Introduction}

In Europe, at least half of the elderly population who suffer from dementia, have mild form of dementia 24. Elderly citizens who are suffering from any form of dementia, are increasingly dependent on their social environment. Although people in early stages of dementia, are often able to perform everyday tasks without help. Their dependence on caregivers and need for assistance increases as the dementia syndrome progresses. This dependency puts strains on both the patients and their caregivers. As a patient's dependency on caregivers grows, patients feel reluctant to leave their home for the fear of getting lost, losing track 
of time or forgetting their goals. While the person with dementia is outside on his own, caregivers are burdened by worrying for the patients' safety.

In sum, during the course of the dementia syndrome, patients are likely to be less autonomous. They increasingly depend on caregivers with respect to their everyday tasks and activities. Most of the cases, in the early or mild stages of dementia, the caregiver is a family member or friend [30], thus part of the informal social network of the patient. A solution to this problem might both increase patients independence and relieve caregivers from (parts of) the stress they experience while taking care of their relative with dementia. A potential solution might be found in the realm of virtual connectedness to the social context.

In this paper we show how to map two of the main issues with dementia on the design of a mobile interactive systems. Roughly, people with dementia suffer from being lost in time and lost in space. Space and time are two important aspects that are taken into account in typical context-aware user interfaces 101 . Besides these typical contextual parameters, the social network itself is also considered as part of the context in our approach. This implies that these contextual parameters (time, space and social network) should be part of the design process for building an interactive systems for people with dementia.

In order to validate our concept, we have developed a prototype of an interactive system, which is an aiding tool for persons with dementia. The prototype was built taking into account the important factors for independence of persons with dementia as mentioned above time, location and social network of a person with dementia to provide context aware help. It offers help only when it is necessary, without patronizing the user all the time.

\section{Background}

\subsection{Dementia}

Dementia is a term for a syndrome related to the loss of cognitive functions. It is usually the result of conditions that cause dysfunction of the brain. Draper [12] defines the dementia syndrome as an acquired decline in memory and thinking (cognition) due to brain disease that results in significant impairment of personal, social or occupational function. He explains that other brain functions such as orientation, language, reasoning and language processing are also affected. Dementia progresses over time. However the progression of dementia is individual and different brain functions do not decline at the same rate, some symptoms develop later in the course of the disease than others. The progression of dementia can be roughly classified according to the degree of severity. For example, by applying the scale developed by Leonard Berg [20. The focus of this paper is on questionable and mild dementia. Further stages require increasing personal caring, which is outside the scope of our work. Dementia also puts enormous strains on the patients social context, especially as dementia progresses [12]. In most cases, the caregivers are spouses and when they can no longer care for the patient, their children take over 30. In a comparative study between caregivers 
for dementia and caregivers for non-dementia people, Ory et al. 26] found that the former was in almost all aspects more demanding than the latter.

\subsection{Assistive Social Navigation}

As mentioned earlier a person with dementia needs help with memory, spatial and temporal orientation. A context aware reminder and navigation system can help to partly regain independence by providing focused navigation information when necessary.

People often make decisions about their actions based on what other people have done in the past or on what other people have recommended them. This process is referred to as everyday social navigation [11. When people need clue about some actions or things, they mostly base their decisions and actions on recommendations by people they know or people who share at least some common ground (e.g. person buying a book may also want to see what other people bought, who bought the same book). The process of finding the required information source and identifying the relevant information requires a lot of effort when it has to be performed in situ. An alternative method is to gather information by asking other people for advice [21]. Identifying the appropriate person for answering questions in a particular context and in suitable time is not a straightforward task [19], it is difficult even for people who do not suffer from dementia.

This becomes more difficult for a person with dementia, especially when he is spatially or temporally disoriented. Because of the disorientation, he becomes frustrated and it gets more difficult for him to formulate his enquiry. Assistive social navigation is tailor made information provided as suggestion for a particular end user. The suggestion is produced by a familiar person e.g. caregiver. In our case, the end user is the elderly person with dementia who needs information or help to complete any tasks by himself. When a person with dementia is lost in time or space (e.g. forgets where he is, where he is going or what he is supposed to do), he usually cannot get back to his normal state immediately. A person with dementia is often aware that he is lost, or he forgot his goal [29]. In this kind of frustrating situation he is unable to carry out his normal activity. Often he can recall his goal with some clues. But only his informal caregiver (e.g. spouse), who is aware about him and his tasks, can easily provide that targeted clues. Thus the caregiver can assist the person with dementia to navigate to complete his tasks only when it is needed; maintaining his autonomy when he is not disoriented.

\subsection{Technologies for Dementia Aid}

The need for independence for both people with dementia and their caregivers is commonly agreed on by researchers in the field [31|2914]. Mulvenna et al. 24] conducted a study to analyze the need of people with dementia and their caregivers. They chartered the needs for more independence by means of memory 
assistance and by providing more context such as spatial and temporal orientation, social contact and, social interaction. The person(s) who are in early stages or in mild stages of dementia are capable of living independently, and have occasional need for help [2]. A context-aware system can exert such support without patronizing the user. Based on these findings, the context-of-use will be explicitly taken into account in our approach to create an interactive systems that prolog independent living of elderly people with early and mild dementia.

Over the last decade, numerous IT-based support system for elderly people with dementia have been proposed [22,17/29]. One of the main objectives of those was to ensure helping elderly people live a safe and independent life. Providing cognitive support as a way of improving quality of life in general [16 has also been proposed. There are different ways in which such system offered support for independent living in some extent such as, tele-monitoring, reminding important activities [13, such as time to take medication etc. Reminder is a form of usually short message that helps people remember what is to be done. It consists of two independent features, signal and description [28. An alarm clock could be a signal only reminder whereas an email notification is both visual signal and text description of the task to be done. Dey et. al. 9] proposed context-aware reminder system, other reminder system taking into account time, location, user present in the vicinity, user's activity.

Robinson et al. 29] developed a prototype to facilitate independent living for patients with light dementia that includes a communication platform for patients with dementia and their families. Hettinga et al. [16] evaluated the safety of navigation aid for people with mild dementia. The authors conclude that there is no evidence suggesting that the use of navigation devices for people with mild dementia are unsafe. Miskelly used a tracking system to define safe zones for persons with dementia. When the dementia person left the safe zone, the caregiver would receive a notification with name of person, location and cause 23 . Social awareness has been studied to enrich context information to provide context aware and appropriate help for nomadic persons [195], and limited social awareness such phone book with the picture of social contact for persons with light or mild dementia has been proposed [8]. In our approach, we combine navigation aid and tracking system along with reminder service and social awareness as a mediator, to provide a tool to plan and assist activities of daily living to regain independence.

\section{Scenario}

This section motivates the use of an assistive application for a person with dementia. We use the COMuICSer approach presented by Haesen et al. in [15]. This approach uses a storyboard to reveal important information on the context of the user and helps to elicit the requirements to be taken into account. Furthermore, based on [18] it provides us with context information for usage in other models instantaneously. 
A storyboard illustrating a regular day for a person with mild dementia is shown in Fig. 1] The storyboard depicts the sequence of activities that the person with dementia performed during that day. At one point, the person with dementia loses his orientation and cannot perform his task without help. At this point, the storyboard introduces other roles, such as a caregiver, that support the target user of our system. This information will be fed in the construction of the social network underpinning the target application.

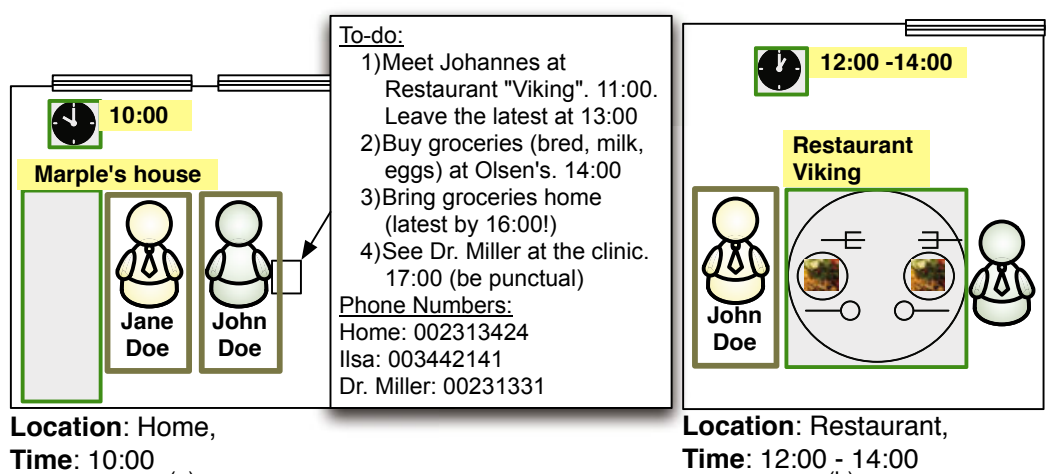

(a)

(b)

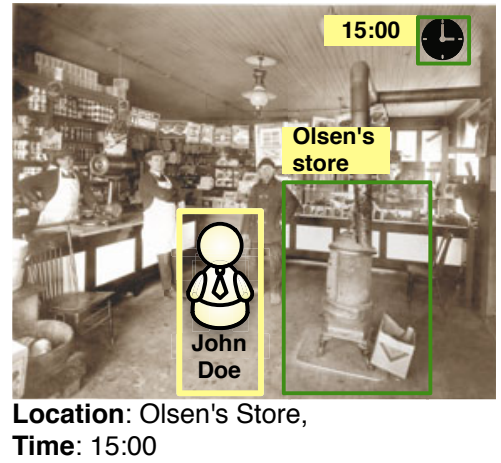

(c)

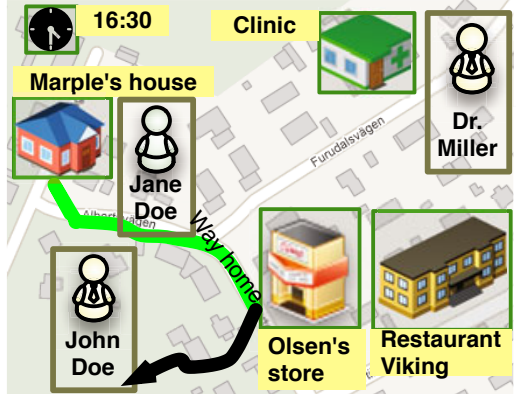

Location: In the woods,

Time: 16:30

(d)

Fig. 1. A day in Mr. John Doe's life, a) Jane helps John to plan his day, b) John meets his friend at the restaurant, c) John buys groceries and d) John is lost

The depicted scenario of Fig. 1 goes as follows: John is in his early seventies, married to Jane. They have been living in a small village for more than three decades. Their daughter Ilsa is married and lives in the same village. John is a retired school teacher. He is an enthusiastic bird watcher and spends a lot of his time on this hobby. Since his retirement, he enjoys reading books and hiking. Lately he started forgetting things but managed to hide it from Jane by taking notes or blaming it on others. But now he started having difficulties to find his 
way back home. This happens even when he is in familiar places he has known for years. Initially he took this very lightly and did not pay attention. Until one day it took John an exceptionally long time to arrive back home; he was lost and came back with the help of nomadic foresters. Jane became highly worried after this incident. She started watching her husband more closely and noticed that her husband forgets small things on a regular basis as well. After talking to the family doctor and friend, Dr. Miller, he was diagnosed as having early stage of dementia. This diagnosis was made two months ago. Since then, John has been quite sad. He often worries about his future and gets angry a lot when he cannot remember things.

Today, John planned to go to local restaurant, and to the grocery shop afterwards. Next, he will come back home. In the late afternoon at 5PM he is going to the see Dr. Miller. In the morning, Jane made a simple to-do list on paper. She also wrote their home phone number, the phone number of their daughter Ilsa and the phone number of Dr. Miller, in case she cannot be reached. She wanted to make sure that John does not forget all his tasks that he wanted to perform, and that he does not feel afraid of getting lost again. It was already after 4PM and John did not come back home yet (shown in the last frame of Fig. 1). Jane started worrying, should she call him to check everything is OK and remind him where he needed to go?

Besides having the sequence of actions crystallized and overview of the context of use by using the COMuICSer approach, it also provides support for describing the personas involved in the scenario. Next in our approach, these personas will be converted into roles in the user interface. The personas can also be used as filters when integrating the social network, i.e. when the patient requires support from a specific type of caregiver the persona can be used as an indication for this type. In our scenario a doctor acts as the caregiver in the depicted situation.

\subsection{Elicited Requirements}

The scenario presented in the previous section describes the current situation and reveals the requirements for an interactive system for persons with dementia. We want to emphasize the following context of use especially plays an important role for our target application:

- the dementia person's time and location

- the caregivers' time and location

- the social context and the participants' states, e.g. a caregiver is busy or available

Time and location deserve special attention, since these are two parts of the context of use that a person with dementia has the most difficulties with. This means a mismatch between the time and location of the person with dementia with the time and location on which a specific task should be executed needs to be explicitly handled by the resulting interactive system. A task and dialog model provide more insight in how the system can actually support the user. 
The scenario further hints at the computing devices and communication services that should be available to support an improved scenario. For our purpose, a smartphone is sufficient to support the tasks and activities at hand.

\section{Approach}

\subsection{User Interface Design Considerations for People with Dementia}

Based on the scenario outlined in the previous section and related work in Sect. 2.3. it is clear that the design of an interactive system for elderly people with symptoms of early or mild dementia poses a number of challenges. Besides typical physical age-related impairments, such as reduced eye sight, decreasing motor skills or hearing difficulties, dementia adds further requirements to the interface design. The deteriorating short term memory, spatial and temporal orientation and the increasing difficulties with performing complex tasks make traditional user interfaces very difficult to use for dementia patients. Newell et al. 25] make several recommendations for the design of information technology for people with cognitive impairments. These are classified based on the type of impairment:

- Mitigate memory impairment. The interface should be simple and limit the possibilities for error. Users should be able to recognize errors and correct them. The system can assist by providing feedback and asking, where appropriate, the user to confirm an action and offer sensible reminders and prompts. Additionally, the user interface can assist the user's memory by providing navigation in the interaction.

- Avoid cognitive overload. The interface should limit options and be simple. Whenever possible, the dialog flow should be linear and parallel tasks possibly avoided.

- Take into account individual characteristics of dementia. Systems for patients with cognitive impairments must be adaptable to the patient's personal conditions that change over time.

The first two guidelines are appropriate for every end user and are especially desirable for people with dementia. Due to their cognitive decline, persons with dementia have difficulties performing parallel tasks and recalling the flow of task even if they are familiar with the task.

Context-awareness is a key feature to achieve personalized, situation-aware adaption of services, which is in accordance with the guidelines described above. Context-aware interactive systems are able to adapt according to the situational context in which they are executed. The context includes characteristics of the user, the device, the environment or a combination of these. Context can be used as input in the design process (for the parts that are known beforehand) and processed during runtime usage of an interactive system 6. The latter ensures adaptability of the system while it is being used. 


\subsection{A Model Driven Design Approach}

As appropriate handling of context is crucial to our application, we explored ways to effectively take all the relevant context influences into account from the start of the development process. Task models, such as the ConcurTaskTree (CTT) 27, are established starting points in the development process of interactive systems, such as the reference framework proposed by [6. We explored the usage of the Contextual ConcurTaskTrees 4. This allowed us to effectively represent influences of context changes on the tasks. However, the high-level of abstraction and the restricted set of information proved too limited to effectively create the application.

We thus looked for a more concrete notation that helped in clarifying the overall picture. Robinson et al. 29] developed applications for people with dementia and suggest eliciting the users requirement into the design by using storyboards. This improves the involvement of all stakeholders in the elicitation process. A storyboard is also a useful tool to capture the context of use. It clearly helps to highlight the social context of the persons who are communicating.

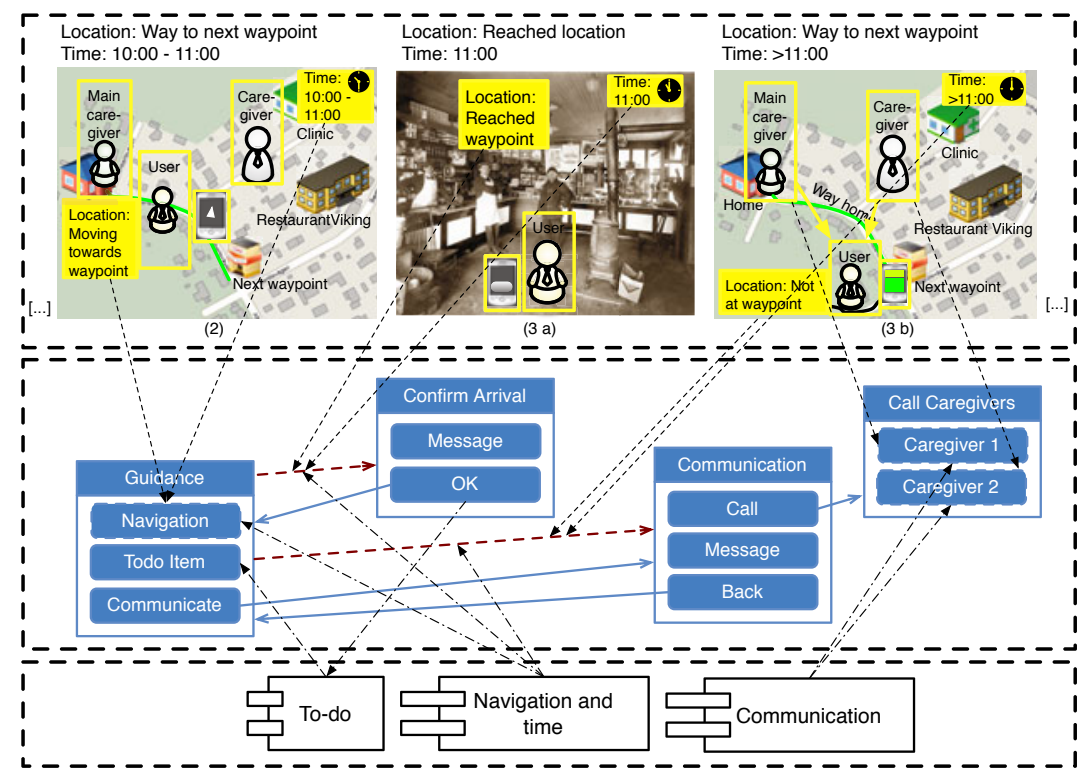

Fig. 2. Relation between the models used to develop the system. On the top, part of the storyboard showing the actors and their context. In the middle, the dialog model and arrows pointing the involvement of context and at the bottom, application model arrows showing influence on the dialog and the presentation model.

Section 3.1 and 4.1 discussed the requirements for the interactive system for a person with dementia. A storyboard is drawn that described the person with 
dementia and the caregivers using the system that fulfills the requirements in Sect. 3.1. The storyboard shows a sequence of scenes depicting the situations and context in which the interactive system needs to operate. A scene shows the person with dementia and his mobile device and other (secondary) users (e.g. caregivers). We also add the time and place of a scene. Each scene is also accompanied by a textual description that further specifies the details. We used the COMuICSer tool [15] to annotate important characteristics in the storyboard (e.g. actors, location, time) and textual scene description.

The storyboard depicts the caregivers and the location and time when the user performs an activity. For example, one scene shows the user visiting the doctor at a certain time and place. The last scene shows the person with dementia getting lost. This scene hints at an incident were the person with dementia may require help. The context information time and location are associated with events that occur when certain predefined context rules are triggered. For example, the user is not at a given location at a predefined time or the user has reached the location for this task on time. The specific rules that are triggered by this context information are discussed into detail in Sect. 5.5. We further used low fidelity prototyping by sketching the user interfaces that would be shown to the user in the different scenes of the storyboard.

Based on the specifications captured by the storyboard (top of Fig. 2), we defined a dialog model (middle of Fig. 2) that describes the behavior of the application. The sequence of scenes indicates the states in the dialog model and how they are connected. The visual nature of the COMuICSer storyboard allows to easily identify all aspects of the context that are relevant for each state in the dialog model. Context events that are related to time and location trigger affect the flow of the dialog. The dotted arrows in Fig. 2 indicate this influence. In contrast with typical behavior of context-aware systems, the system does not adapt its presentation according to the context of use but tries to figure out how it can help the user to accomplish the tasks at hand. This means the context is not used to automate the system but rather the system works in a mixed initiative approach, the goal is to empower the user by pro-actively providing information cues and making suggestions.

Next, we need to create the presentation that exposes the behavior described in the previous paragraph. First, we have the presentation based on the sketches done in the earlier stage of the development. The presentation model and dialog model link is established by sketching the interfaces for the different scenes and clearly relating them with each other. There is also a link between the social context and the presentation model that needs to be taken into account. The availability states of caregivers are represented differently; we chose distinct colors for that purpose.

In the last step, the components of the system and their functionality are defined. This is taken as well from the requirements captured at the start of the development process. We identified a component for the to-do list manager, the social network, a component for location-based events and for time-based events. These components are described with the application model (bottom of Fig. 2). 


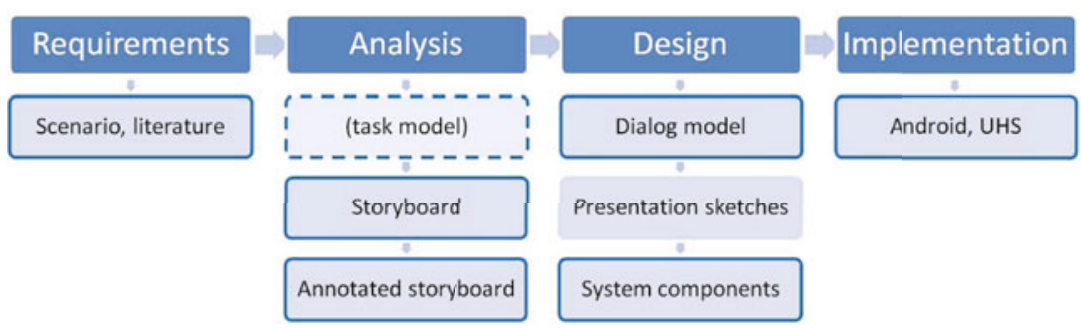

Fig. 3. The development process, fine grained steps in which context, and context influences are explicitly described have borders with full lines

The application model is then linked to the dialog and presentation model for orchestrating the behavior of the overall system.

The links between the application model and the dialog model are bi-directional: from the dialog model, functions described in the application model can be called. Dialog changes that are initiated by the application logic are triggered through time and location related events. Section 5.5 provides more details about these events. The link between the application model and presentation model determines how objects that are returned by an application are displayed. For example, depending on the availability status, a caregiver is displayed differently.

Figure 3 summarizes the different steps taken to develop our application. It clearly shows the mix informal and abstract formal notations during the development process and the explicit definition of context (influences) in almost all steps from the start of the development process. The task model step has a dashed border because the information it provided was not complete enough and could be completely replaced by the storyboard.

\section{The Resulting System}

\subsection{Overview}

Our system consists of three main building blocks: the user interface, context rules and the application logic. The user interface layer consists of three parts, namely 1) the to-do list and navigation module that is used by the person with dementia, 2) The to-do list manager that is the interface for the primary caregiver to manage the to-do list of the person with dementia and 3) the communication module that connects the person with dementia with the caregivers. The application logic consists of 1) the context module that receives time and location information, 2) the to-do list repository that stores the plans for the person with dementia and 3) the social network that connects the person with dementia with his or her social context. Between the user interface and the application logic reside the context rules. The rules use the current time and location, and assigned time and location for the current task to change the dialog. 


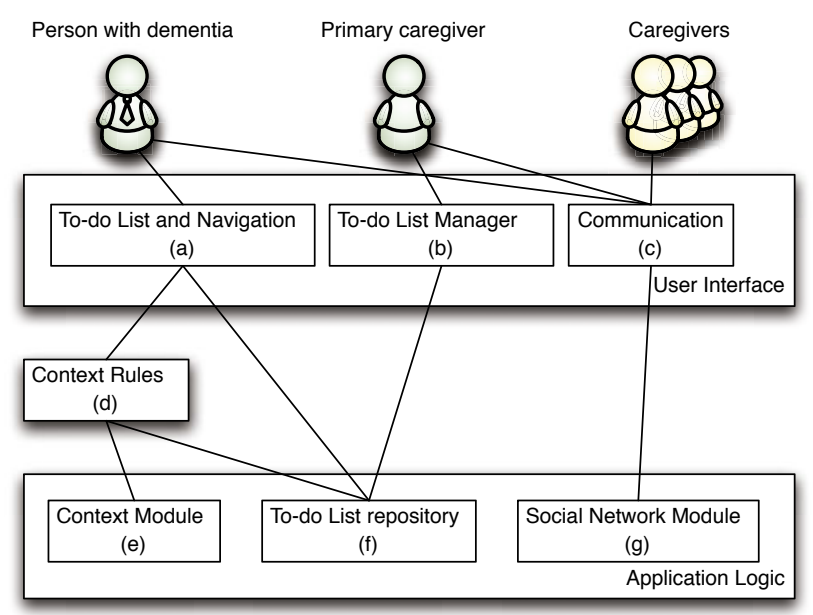

Fig. 4. System overview

The system runs on different communicating devices: the person with dementia uses a smart phone that runs Google Android. The communicating caregivers can use any Java enabled communicating devices that can connect to the phone network and the Internet. In the prototype described in this paper, the behavior of the system is presented in Java. The description of the user interface presentation uses the native XML user interface description language of the Google Android platform.

\subsection{To-Do List and Navigation}

The to-do list and navigation (see Fig. 4(a)) is used by the person with dementia to navigate through his plan. It receives the plan from the to-do list repository. The main navigation display shows information about the current task that is relevant to the user. This is an arrow indicating the direction where the user is expected to go, the name and time of the current task and a button that takes switches to the communication interface. An example of the to-do list and navigation interface is shown in Fig. 5(b). The to-do list and navigation module receives the location information from the context module through the context rules. This corresponds to the linking between the dialog model and the application model (see Fig. 2). The rules initiate intra and inter-dialog changes in response to the current time and location, and time and location associated with the current task.

\subsection{To-Do List Manager}

The to-do list manager (see Fig. 4(b)) is used by the primary caregiver, in most cases the spouse, to compile a plan for person with dementia. A plan is a set 
of tasks that is uploaded to the to-do list repository which is accessed by the to-do list navigator of the person with dementia. For each new task the caregiver defines the name and description of the task, the time and location associated with this task and possible relevant caregivers (e.g. daughter, brother, personal physician). To avoid confusing the user, the list of the caregivers defined at this stage are always displayed in the communication screen (see 5(c).

\subsection{Communication Module}

The communication module (see Fig. 4(c)) offers a communication channel between the person with dementia and his caregivers. The person with dementia can choose between selecting predefined text messages or making a phone call. The list of available caregivers is provided during run-time via an XML-RPC connection from the social network module, discussed in Sect. 5.7. The caregivers are marked in a color that depicts their availability status. This is shown in Fig. 5(c), It also contains an option to return to the navigation screen.

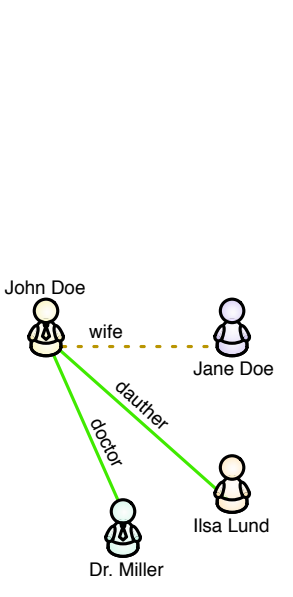

(a)

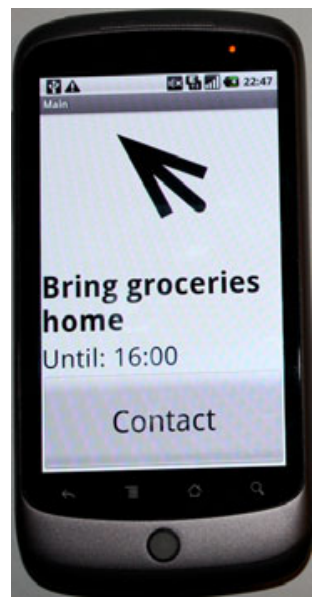

(b)

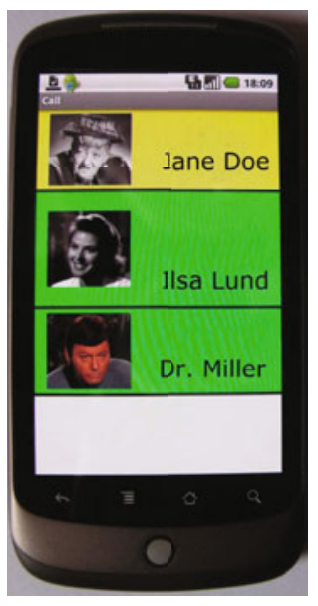

(c)

Fig. 5. The system in action, 5(a) visualization of the social network, dashed line represents unavailable user 5(b) navigation screen showing the navigation arrow pointing towards the location of the current task to perform, as well as information about the task and 5(c) communication screen showing list of caregivers, their availability status is distinguished by colors

\subsection{Context Module and Context Rules}

The context module (see Fig. 4(e)) listens for time and location information. These are passed to the context rules (see Fig. 4(d)). The context module is implemented as a location listener that is registered with the the Google Android 
platform Location Manager to receive location updates. It further contains a local thread that queries the current time in predefined intervals. The context rules are executed after each time interval.

Context rules mediate between the context events sent by the context module, the current task and the dialog model. Based on time and location, intra or interdialog changes can occur.

- Intra-dialog changes occur for example when the user has reached the location for the task but is early or when the user is on time. In this case, the person with dementia is notified with an alert that pops up on the screen.

- Inter-dialog changes are triggered when a disorientation of the user is suspected. This is measured when the user has not reached the location for the task at the expected time. In this case, the user interface is changed to the communication screen. The user can at any time switch back to the to-do list and navigation screen.

\subsection{To-Do List Repository}

The to-do list repository is a database that stores the lists of tasks that the person with dementia wants to perform. This database is accessed by the primary caregiver to create the to-do list (i.e. the plan). The rest of the time, it is accessed by the to-do list and navigation module to inform the user about the current task.

\subsection{Social Network Module}

The social networking module exploits the Ubiquitous Help System (UHS) [19] as a basis for the social networking communication platform. The UHS is based on a client-server architecture. An HTTP/XML based communication framework is used to facilitate a UHS client to communicate with other UHS clients. The UHS client can send and receive plain text and attached files such as a regular email. When a user needs help and asks query, the controller of the UHS sends the query to other clients via server. The controller of the receiving client initiates processing for profile and availability status matching, and replies with the appropriate information.

In the prototype we developed, a user is identified by a Friend-Of-A-Friend (FOAF) profile. This contains information about the social relations of the user and traditional information that identifies the user (such as name, address). FOAF is a Resource Description Framework vocabulary (RDF) [3] for describing people and social networks.

\section{Discussion}

In this paper we presented an interactive system and showed that applications for people with dementia can be created by explicitly taking context into account in the design process. In our approach, context dependencies are defined in an early 
stage in the development process. We utilized the COMuICSer storyboarding tool to elicit our design requirements. This tool supports annotation of context information (time, location, social context) and proofed to be more informative than task models.

Typical context aware user interfaces consider space and time as important aspects. In addition, our approach includes the social context of the users involved in a communication process. The inclusion of social factors goes further than simply using internal context of the user. We have exploited the Ubiquitous Help System (UHS), introduced in earlier work, to empower social networking feature into our prototype. This completes the different types of context that need to be supported for applications that target people with early-stage dementia.

However, in our current prototype, the interaction from the caregivers' perspective has not been fully explored yet. Our aim was mainly to present an appropriate approach that addresses the three different types of context (location, time, social) that are important for these users explicitly during the development of such a system. In our future work, this issue will be addressed in order to have a system, which can be tested with real users.

The development of this prototype learned us that informal notations play an important role in the development of context-sensitive interactive systems, such as the one described in this paper. The explicit relations between the informal description and the formal models, such as the dialog model, inspire us to explore potential automation or consistency checks between the informal specifications offered by the scenario model and the presentation sketches, especially since there are already existing formalizations of both notations [187.

\section{Acknowledgments}

The authors are pleased to thank Mieke Haesen of EDM for her help in utilizing the storyboard 1 tool. Part of the research at EDM is funded by EFRO (European Fund for Regional Development) and the Flemish Government. Funding for this research is also provided by the Research Foundation - Flanders (F.W.O. Vlaanderen, project CoLaSUE, number G.0439.08N) and one of the authors is funded by the Hasler Foundation (LoCa project).

\section{References}

1. Balme, L., Demeure, A., Barralon, N., Coutaz, J., Calvary, G.: CAMELEON-RT: A software Architecture Reference Model for Distributed, Migratable, and Plastic user Interfaces. Ambient Intelligence 3295, 291-302 (2004)

2. Baumgarten, M., Mulvenna, M.: The Role of Context-aware Computing in Support of People with Dementia. In: Supporting People with Dementia Using Pervasive Health Technologies, pp. 131-143. Springer, Heidelberg (2010)

\footnotetext{
${ }^{1}$ StoryBoardML: http://research.edm.uhasselt.be/ kris/research/projects/
} StoryBoardML/storyboards.html 
3. Beckett, D., McBride, B.: RDF/XML Syntax Specification (Revised). Tech. rep., W3C (February 2004), http://www.w3.org/TR/rdf-syntax-grammar/

4. Van den Bergh, J., Coninx, K.: Contextual concurtasktrees: Integrating dynamic contexts in task based design. In: PerCom Workshops, pp. 13-17. IEEE Computer Society, Los Alamitos (2004)

5. Bilandzic, M., Foth, M., De Luca, A.: CityFlocks: designing social navigation for urban mobile information systems. In: DIS 2008: Proceedings of the 7th ACM Conference on Designing Interactive Systems, pp. 174-183. ACM Press, New York (2008)

6. Calvary, G., Coutaz, J., Thevenin, D., Limbourg, Q., Bouillon, L., Vanderdonckt, J.: A unifying reference framework for multi-target user interfaces. Interacting with Computers 15(3), 289-308 (2003)

7. Coyette, A., Kieffer, S., Vanderdonckt, J.: Multi-fidelity Prototyping of User Interfaces. In: Baranauskas, M.C.C., Palanque, P.A., Abascal, J., Barbosa, S.D.J. (eds.) INTERACT 2007. LNCS, vol. 4662, pp. 150-164. Springer, Heidelberg (2007)

8. Davis, R., Nugent, C.D., Donnelly, M.: Prototyping Cognitive Prosthetics for People with Dementia. In: Mulvenna, M.D., Nugent, C.D. (eds.) Supporting People with Dementia Using Pervasive Health Technologies, pp. 145-163. Springer, Heidelberg (2010)

9. Dey, A.K., Abowd, G.D.: CybreMinder: A Context-Aware System for Supporting Reminders. In: Thomas, P., Gellersen, H.-W. (eds.) HUC 2000. LNCS, vol. 1927, pp. 172-186. Springer, Heidelberg (2000)

10. Dey, A.K., Häkkilä, J.: Context-Awareness and Mobile Devices. User Interface Design and Evaluation for Mobile Technology 1, 205-217 (2008)

11. Dourish, P., Matthew, C.: Running out of space: Models of information navigation. Presented at HCI 1994, Cambridge, UK, NA, p. 134 (1994)

12. Draper, B.: Dealing with dementia: a guide to Alzheimer's disease and other dementias / Brian Draper. Allen \& Unwin, Crows Nest (2004)

13. Du, K., Zhang, D., Zhou, X., Mokhtari, M., Hariz, M., Qin, W.: HYCARE: A Hybrid Context-Aware Reminding Framework for Elders with Mild Dementia. In: Helal, S., Mitra, S., Wong, J., Chang, C.K., Mokhtari, M. (eds.) ICOST 2008. LNCS, vol. 5120, pp. 9-17. Springer, Heidelberg (2008)

14. Graff, M., Vernooij-Dassen, M., Thijssen, M., Dekker, J., Hoefnagels, W., Rikkert, M.: Community based occupational therapy for patients with dementia and their care givers: randomised controlled trial. British Medical Journal 333(7580), 1196 (2006)

15. Haesen, M., Luyten, K., Coninx, K.: Get Your Requirements Straight: Storyboarding Revisited. In: Gross, T., Gulliksen, J., Kotzé, P., Oestreicher, L., Palanque, P., Prates, R.O., Winckler, M. (eds.) INTERACT 2009. LNCS, vol. 5727, pp. 546-549. Springer, Heidelberg (2009)

16. Hettinga, M., De Boer, J., Goldberg, E., Moelaert, F.: Navigation for People with Mild Dementia. Studies in Health Technology and Informatics 150, 428 (2009)

17. Lauriks, S., Reinersmann, A., Roest, H., Meiland, F., Davies, R., Moelaert, F., Mulvenna, M., Nugent, C., Dröes, R.: Review of ICT-Based Services for Identified Unmet Needs in People with Dementia. In: Mulvenna, M.D., Nugent, C.D. (eds.) Supporting People with Dementia Using Pervasive Health Technologies, Springer, Heidelberg (2010)

18. Luyten, K., Haesen, M., Ostrowski, D., Coninx, K., Degrandsart, S., Demeyer, S.: Storyboard creation as an entrypoint for model-based interface development with UsiXML. In: UsiXML, pp. 1-8 (2010) 
19. Mahmud, N., Luyten, K., Coninx, K.: Context Aware Help and Guidance for LargeScale Public Spaces. In: SMAP 2009: Proceedings of the 2009 Fourth International Workshop on Semantic Media Adaptation and Personalization, pp. 105-110. IEEE Computer Society Press, Washington (2009)

20. Maj, M., Sartorius, N.: Dementia. WPA series, vol. 3. John Wiley and Sons, Chichester (2003)

21. McDonald, D.W., Ackerman, M.S.: Expertise recommender: A flexible recommendation system and architecture. In: Proceedings of the 2000 ACM Conference on Computer Supported Cooperative Work, pp. 231-240. ACM, New York (2000)

22. Mileo, A., Merico, D., Bisiani, R.: Support for context-aware monitoring in home healthcare. J. Ambient Intell. Smart Environ. 2(1), 49-66 (2010)

23. Miskelly, F.: A novel system of electronic tagging in patients with dementia and wandering. Age and Ageing 33(3), 304 (2004)

24. Mulvenna, M.D., Nugent, C.D., Moelaert, F., Craig, D., Dröes, R.: Supporting People with Dementia Using Pervasive Healthcare Technologies. In: Advanced Information and Knowledge Processing, pp. 3-14. Springer, London (2010)

25. Newell, A., Carmichael, A., Gregor, P., Alm, N., Waller, A.: Information technology for cognitive support. In: Sears, A., Jacko, J.A. (eds.) The Human- Computer Interaction Handbook: Fundamentals, Evolving Technologies, and Emerging Applications, ch. 41, 2nd edn., pp. 811-828. CRC Press, Boca Raton (2007)

26. Ory, M., Hoffman III, R., Yee, J., Tennstedt, S., Schulz, R.: Prevalence and impact of caregiving: A detailed comparison between dementia and nondementia caregivers. The Gerontologist 39(2), 177 (1999)

27. Paterno, F.: Model-Based Design and Evaluation of Interactive Applications (Applied Computing). Springer, Heidelberg (2000)

28. Ren, Y., Kiesler, S., Fussell, S.: Multiple group coordination in complex and dynamic task environments: Interruptions, coping mechanisms, and technology recommendations. J. Manage. Inf. Syst. 25(1), 105-130 (2008)

29. Robinson, L., Brittain, K., Lindsay, S., Jackson, D., Olivier, P.: Keeping In Touch Everyday (KITE) project: developing assistive technologies with people with dementia and their carers to promote independence. International Psychogeriatrics 21(03), 494-502 (2009)

30. Schulz, R., Martire, L.: Family caregiving of persons with dementia: prevalence, health effects, and support strategies. American Journal of Geriatric Psych. 12(3), 240 (2004)

31. Woods, B.: Promoting well-being and independence for people with dementia. International Journal of Geriatric Psychiatry 14(2), 97-105 (1999) 